\title{
Healthy Eating: Lower Your Blood Pressure with DASH ${ }^{1}$
}

Valerie Weyenberg, Karla P. Shelnutt, and Linda B. Bobroff ${ }^{2}$

About one third of American adults have high blood pressure. Uncontrolled high blood pressure can cause heart disease, kidney disease, stroke, and blindness. Being physically active, taking medications as directed, and eating a healthful diet all help to control blood pressure. One eating plan that has been shown to prevent and reduce high blood pressure is DASH. Read on to learn how the DASH eating plan can help you lower your blood pressure.

\section{What is the DASH eating plan and why is it important?}

DASH stands for Dietary Approaches to Stop Hypertension. The DASH eating plan is rich in fruits, vegetables, and low-fat dairy products, which makes it high in calcium, magnesium, and potassium - three minerals that lower blood pressure. It is low in saturated fat and cholesterol, and moderate in total fat. The eating plan is more effective when you also reduce your sodium intake.

Several studies have shown that the DASH diet lowers blood pressure in people with high blood pressure. It also helps maintain normal blood pressure. Eating the DASH way can help you lose weight if you are overweight. Losing excess body weight can help lower your blood pressure.

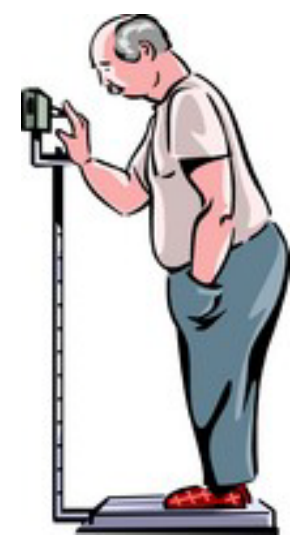

\section{How much and what types of foods should I eat?}

The DASH eating plan includes lots of fruits, vegetables, low-fat dairy products, and whole grains. You also will eat poultry, lean meats, fish, legumes, eggs, and nuts.

For a 1600 calorie meal plan, the DASH diet includes the following amounts of food from each food group:

\begin{tabular}{|l|c|}
\hline Food Group & Servings/day \\
\hline $\begin{array}{l}\text { Grains } \\
\text { (most of your servings } \\
\text { should be whole grain) }\end{array}$ & 6 \\
\hline Vegetables & $3-4$ \\
\hline Fruits & 4 \\
\hline Low-fat dairy & $2-3$ \\
\hline $\begin{array}{l}\text { Lean meats, poultry, and } \\
\text { fish }\end{array}$ & $3-6$ \\
\hline Nuts, seeds, or legumes & 3 per week \\
\hline Fats and oils & 2 \\
\hline Sweets & 0 \\
\hline
\end{tabular}

1. This document isFCS8884, one of a series of the Department of Family, Youth and Community Sciences, Florida Cooperative Extension Service, IFAS, University of Florida, Gainesville, FL 32611. First published: October 2009. Please visit the EDIS Web site at http://edis.ifas.ufl.edu.

2. Valerie Weyenberg, MSDI student, Department of Food Science and Human Nutrition; Karla P. Shelnutt, PhD, RD, assistant professor, and Linda B. Bobroff, professor, Department of Family, Youth and Community Sciences; Cooperative Extension Service, Institute of Food and Agricultural Sciences, University of Florida, Gainesville, FL 32611. 
Food group recommendations in the DASH eating plan also are available for 2000, 2600, and 3100 calorie diets and can be found at: http://www.nhlbi.nih.gov/health/public/heart/hbp/dash/new dash.pdf

In general, older adults need fewer calories than younger adults. If you think you need more than 1600 calories each day, you can add more servings.

\section{What is a serving?}

Here are some examples of one serving from each of the food groups:

\section{Grains}

1 slice of bread

$1 / 2$ cup dry cereal

$1 / 2$ cup cooked rice or pasta

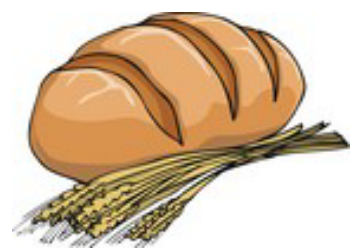

\section{Vegetables}

1 cup raw, leafy vegetables

$1 / 2$ cup cooked or cut-up raw vegetables

$1 / 2$ cup vegetable juice

\section{Fruits}

1 medium fruit

$1 / 4$ cup dried fruit

$1 / 2$ cup frozen, canned or cut-up fresh fruit

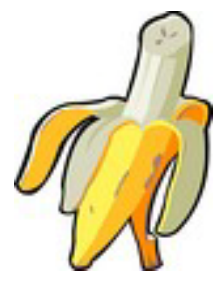

Low-fat dairy (can use fat-free for fewer calories and fat)

1 cup milk or yogurt

1.5 oz cheese

Lean meats, poultry, and fish

1 oz cooked meat, poultry, or fish

1 egg or 2 egg whites

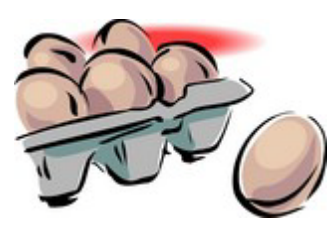

Nuts, seeds, and legumes

$1 / 3$ cup or 1.5 oz nuts

2 Tbsp peanut butter

2 Tbsp or $1 / 2$ ounce seeds

$1 / 2$ cup cooked dry beans

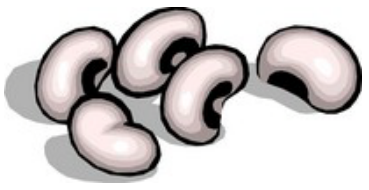

or peas

Fats and Oils

1 tsp margarine or vegetable oil

1 Tbsp mayonnaise

2 Tbsp salad dressing

Sweets

1 Tbsp sugar, jelly, or jam

1 cup lemonade

If switching to the DASH diet sounds overwhelming, don't despair!

Gradually add in the recommended foods such as fruits, vegetables, lowfat dairy, and lean meats. Remember, doing something is better than doing nothing!

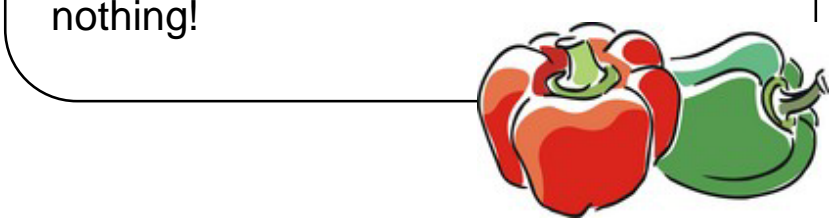

For more information about high blood pressure, see Healthy Living: High Blood Pressure (FCS8599) on the EDIS Web site at: http://edis.ifas.ufl.edu/FY684.

For information on how to make better dietary choices using the food label, read the companion piece Healthy Eating: Understanding the Nutrition Facts Label (FCS8883) at http://edis.ifas.ufl.edu/FY1127. 